\title{
WEAK CONVERGENCE THEOREMS FOR NONEXPANSIVE MAPPINGS AND SEMIGROUPS IN BANACH SPACES WITH OPIAL'S PROPERTY
}

\author{
T. KUCZUMOW
}

\begin{abstract}
Let $X$ be a Banach space with Opial's property, $C$ a weakly compact subset of $X, x \in C$ and $S$ a nonexpansive semigroup on $C$. Then $\{S(t) x\}_{t \geqslant 0}$ converges weakly to a common fixed point of $S$ iff $S(t+h) x-S(t) x \rightarrow 0$ as $t \rightarrow \infty$ for all $h>0$.
\end{abstract}

In this paper we consider a necessary and sufficient condition for the weak (weak-*) convergence of trajectories of nonexpansive mappings and one parameter semigroups of nonexpansive mappings in Banach spaces which satisfy Opial's condition. This problem was studied in $[1,2,5,6,7,9]$.

A Banach space $X$ satisfies Opial's condition [4] for the weak topology if $x_{n} \rightarrow y$ in $X$ implies that

$$
\limsup _{n}\left\|x_{n}-y\right\|<\underset{n}{\lim \sup _{n}\left\|x_{n}-z\right\|}
$$

for all $z \neq y$.

All spaces $l^{p}(1<p<\infty)$ have Opial's property but $L^{p}[0,2 \pi]$ with $1<p \neq 2$ lacks it [4].

A Banach space $X$ satisfies Opial's condition for the weak-* topology if $X$ is a conjugate space to a separable Banach space and for $x_{n} \rightarrow * y$ in $X$ we have

$$
\limsup _{n}\left\|x_{n}-y\right\|<\limsup _{n}\left\|x_{n}-z\right\|
$$

for $z \neq y$.

$l^{1}$ satisfies Opial's condition for the weak-* topology [3]. $l^{\infty}$ does not have Opial's property.

Let $C$ be a subset of a Banach space $X$. A mapping $T: C \rightarrow C$ is said to be nonexpansive if $\|T x-T y\| \leqslant\|x-y\|$ for all $x, y \in C$. A one parameter semigroup $S=\{S(t): t \geqslant 0\}$ of nonexpansive mappings on $C$ is a family of nonexpansive mappings of $C$ into itself satisfying the following conditions:

(i) $S(s+t) x=S(s) S(t) x$ for $s, t \geqslant 0$ and $x \in C$,

Received by the editors July 1, 1983 and, in revised form, January 5, 1984.

1980 Mathematics Subject Classification. Primary 47H10.

Ker words and phrases. Nonexpansive mappings, nonexpansive semigroups, fixed points, Opial's property. 
(ii) $\|S(t) x-S(t) y\| \leqslant\|x-y\|$ for $t \geqslant 0$ and $x, y \in C$.

(iii) $S(0) x=x$ for $x \in C$.

Lemma. Let $C$ be a weakly (weakly-*) compact subset of a Banach space $X$ with Opial's property for the weak (weak-*) topology, $z \in X$ and $\left\{\left\{x_{n}^{m}\right\}: m \in \mathbf{N}\right\}$ be a family of sequences which satisfies the following conditions:

(i) $x_{n}^{m} \in C$ for $n, m \in \mathbf{N}$,

(ii) every $\left\{x_{n}^{m}\right\}$ converges to $y$ in the weak (weak-*) topology,

(iii) $\lim \sup _{n}\left\|x_{n}^{m}-y\right\| \geqslant \lim \sup _{n}\left\|x_{n}^{m+1}-z\right\| \geqslant \lim \sup _{n}\left\|x_{n}^{m+1}-y\right\|$.

Then $y=z$.

PROOF. Let $r_{m}=\lim \sup _{n}\left\|x_{n}^{m}-y\right\|$ for $m \in \mathbf{N}$. Because

$$
C \cap \operatorname{lin}\left\{w \in X: \bigvee_{m, n} w=x_{n}^{m} \vee w=y \vee w=z\right\}
$$

with the weak (weak-*) topology is metrized by some metric $\rho$ [8], then for every $m \geqslant 2$ we may choose $x_{n_{m}}^{m}$ such that

$$
\rho\left(x_{n_{m}}^{m}, y\right) \leqslant 1 / m, \quad\left\|x_{n_{m}}^{m}-y\right\| \geqslant r_{m}-1 / m, \quad\left\|x_{n_{m}}^{m}-z\right\| \leqslant r_{m-1}+1 / m .
$$

Hence we have

$$
\begin{gathered}
x_{n_{m}}^{m} \underset{m}{m} y \quad\left(x_{n_{m}}^{m} \vec{m}_{m}^{*} y\right), \\
\limsup _{m}\left\|x_{n_{m}}^{m}-z\right\|=\lim _{m} r_{m}=\limsup _{m}\left\|x_{n_{m}}^{m}-y\right\|
\end{gathered}
$$

and therefore $y=z$.

THEOREM 1. Let $X$ and $C$ be such as in the Lemma, T: $C \rightarrow C$ be a nonexpansive mapping and $x \in C .\left\{T^{i} x\right\}$ converges weakly (weakly-*) to a fixed point of $T$ iff $T^{i+1} x-T^{i} x \rightarrow_{i} 0\left(T^{i+1} x-T^{i} x \rightarrow_{i} * 0\right)$.

Proof. There exists a subsequence $\left\{T^{i_{n}} x\right\}$ which converges weakly (weakly-*) to $y$. Then for $m \geqslant 1$ we have

$$
T^{i_{n}+m} x_{n} y \quad\left(T^{i_{n}+m} x_{n} * y\right)
$$

and

$$
\begin{aligned}
\underset{n}{\limsup }\left\|T^{i_{n}+m} x-y\right\| & \geqslant \underset{n}{\limsup }\left\|T^{i_{n}+m+1} x-T y\right\| \\
& \geqslant \underset{n}{\limsup }\left\|T^{i_{n}+m+1} x-y\right\| .
\end{aligned}
$$

By the Lemma, $y=T y$ and thus in view of monotonicity of $\left\{\left\|T^{i} x-v\right\|\right\}$ for any $v \in$ Fix $T$, the sequence $\left\{T^{i} x\right\}$ tends weakly to $y\left(T^{i} x \rightarrow_{i}^{*} y\right)$.

THEOREM 2. Let $X$ and $C$ be such as in the Lemma, $S=\{S(t): t \geqslant 0\}$ be $a$ semigroup of nonexpansive mappings on $C$ and let $x \in C$. Then $\{S(t) x\}_{t \geqslant 0}$ converges weakly (weakly-*) to a common fixed point of $S$ iff

$$
\begin{gathered}
S(t+h) x-S(t) x \rightarrow 0 \quad \text { as } t \rightarrow \infty \\
(S(t+h) x-S(t) x \rightarrow * 0 \quad \text { as } t \rightarrow \infty)
\end{gathered}
$$

for all $h>0$. 
Proof. We choose $\left\{t_{n}\right\}$ such that $t_{n} \rightarrow_{n} \infty$ and $S\left(t_{n}\right) x \rightarrow_{n} y\left(S\left(t_{n}\right) x \rightarrow_{n} * y\right)$. For $t>0$ and $m \in \mathbf{N}$ we have

$$
S\left(t_{n}+m t\right) x-S\left(t_{n}\right) \underset{n}{\underset{n}{\rightarrow} 0} \quad\left(S\left(t_{n}+m t\right) x-S\left(t_{n}\right) \underset{n}{x} * 0\right)
$$

and hence

$$
\begin{aligned}
\underset{n}{\limsup }\left\|S\left(t_{n}+m t\right) x-y\right\| & \geqslant \underset{n}{\lim \sup _{n}}\left\|S\left(t_{n}+(m+1) t\right) x-S(t) y\right\| \\
& \geqslant \limsup _{n}\left\|S\left(t_{n}+(m+1) t\right) x-y\right\| .
\end{aligned}
$$

Therefore $y=S(t) y$ for all $t \geqslant 0$ and $S(t) \rightarrow y$ as $t \rightarrow \infty(S(t) \rightarrow * y$ as $t \rightarrow \infty)$.

REMARK. Theorem 2 gives a partial answer to a question raised on p. 550 of [7].

\section{REFERENCES}

1. J. B. Baillon, R. E. Bruck and S. Reich, On the asymptotic behavior of nonexpansive mappings and semigroups in Banach spaces, Houston J. Math. 4 (1978), 1-9.

2. N. Hirano, Nonlinear ergodic theorems and weak convergence theorems, J. Math. Soc. Japan 34 (1982), 35-46.

3. L. A. Karlovitz, On nonexpansive mappings, Proc. Amer. Math. Soc. 55 (1976), 321-325.

4. Z. Opial, Weak convergence of the sequence of successive approximations for nonexpansive mappings, Bull. Amer. Math. Soc. 73 (1967), 591-597.

5. A. Pazy, On the asymptotic behavior of semigroups of nonlinear contractions in Hilbert space, J. Funct. Anal. 27 (1978), 292-307.

6. S. Reich, Weak convergence theorems for nonexpansive mappings in Banach space, J. Math. Anal. Appl. 67 (1979), 274-276.

7. __ A note on the mean ergodic theorem for nonlinear semigroups, J. Math. Anal. Appl. 91 (1983), $547-551$.

8. S. Rolewicz, Metric linear spaces, PWN, Warsaw, 1972.

9. R. Schoeneberg, Weak convergence of trajectories of nonexpansive semigroups in Hilbert space, Israel J. Math. 30 (1978), 130-132.

Instytut Matematyki UMCS, Plac Mari Curie - Skxodowskiej 1, 20 - 031 Lubin, Poland 